\title{
Recurrent PNET with MGMT Methylation Responds to Temozolomide
}

\author{
Jiwon Oh, Juan M. Bilbao, May N. Tsao, Mahmood Fazl, Marie-Christine Guiot, \\ Rolando F. Del Maestro, James R. Perry
}

Can. J. Neurol. Sci. 2009; 36: 654-657

Primitive neuro-ectodermal tumours (PNET) are neuroepithelial neoplasms arising from the germinal matrix of the primitive neural tube. Primitive neuro-ectodermal tumours are predominately a tumour of childhood, accounting for about $20 \%$ of primary central nervous system (CNS) malignancies in children ${ }^{1}$. Typically PNETs occur in the posterior fossa, but supratentorial PNETs in children still account for about $2.8 \%$ of all primary tumours of childhood and adolescence ${ }^{2}$. Much is known about the management of PNETs in children. In adults, on the other hand, supratentorial PNETs are exceedingly rare, with only a handful of cases reported in the current literature. Thus, optimal medical management of supratentorial PNETs in adults remains unclear. Chemotherapy options that have been utilized in previous reports include conventional agents such as vincristine, cisplatinum, cyclophosphamide, procarbazine, and $\mathrm{CCNU}^{2,3}$, in addition to recent reports of successful treatment with temozolomide ${ }^{1,4}$. Here, we present the case of a patient with a supratentorial PNET diagnosed in adulthood, with tumour recurrence on carboplatin and etoposide, which responded to subsequent treatment with temozolomide.

\section{Case Report}

A 42-year-old man presented to the ED with a few weeks history of headache, confusion, nausea, vomiting, and progressive left-sided hemiparesis. An magnetic resonance image (MRI)-head showed a $6.3 \times 5.2 \times 5.0 \mathrm{~cm}$ partially cystic enhancing mass in the right basal ganglia, with mild surrounding edema and mass effect causing a midline shift of seven $\mathrm{mm}$ and right lateral ventricle effacement.

He subsequently underwent craniotomy and gross total excision of the tumour. Clinically, he improved significantly after the surgery and had minimal residual deficits.

Pathology showed a highly cellular tumour composed of poorly differentiated small round blue cells forming pseudorosettes around vessels. The nuclei were pleomorphic with numerous mitoses and apoptotic bodies, with no necrosis (Figure 1). Immunohistochemistry was positive for synaptophysin and negative for CD45, LMWK, HMWK and GFAP, NF, SMI31 and NeuN. These results were deemed consistent with PNET, but a metastatic neuro-endocrine tumour could not be excluded.

He went on to have further imaging, including a computed tompogram (CT) of the chest, abdomen, and pelvis, which did not reveal any findings suggestive of a primary malignancy. Routine staging investigations for radiotherapy included further imaging of his spine and cerebrospinal fluid (CSF) studies to

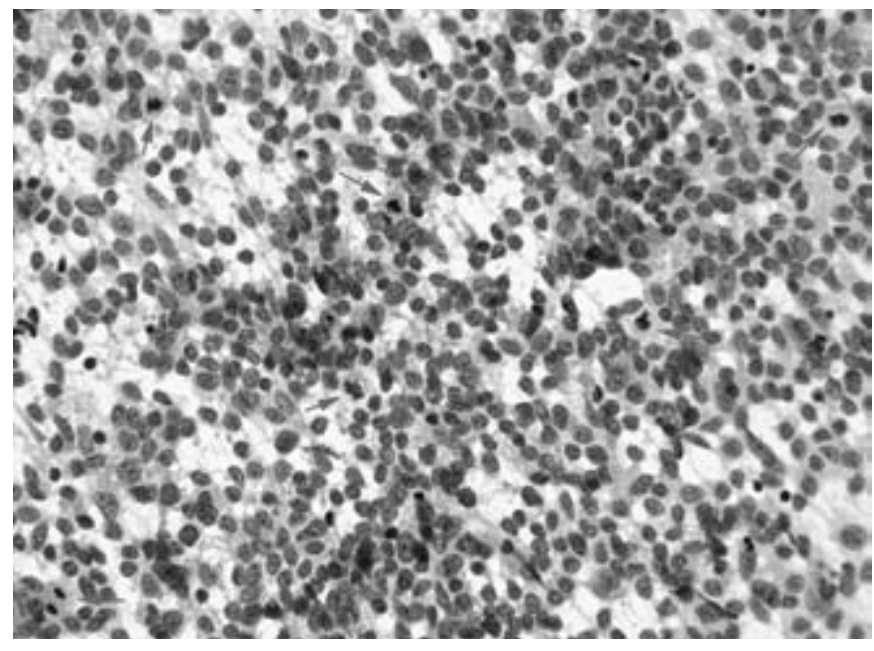

Figure 1: Touch-smear preparation showing an anaplastic round blue cell tumor.

assess for drop metastases. The MRI-spine showed some evidence of degenerative changes in the cervical and thoracic spines, but no evidence of leptomeningeal enhancement. However, in the CSF, there was evidence of malignant cells with two atypical groups of cells with a moderate amount of cytoplasm and irregular nuclei present. He subsequently underwent external beam and cranio-spinal radiation therapy with minimal complications. A follow-up MRI-head showed no evidence of recurrent disease. He was monitored with serial imaging studies.

Nine months after completing radiotherapy, an MRI-head showed a new area of nodular enhancement in the resection

From the Divison of Neurology (JO, JRP), Departments of Medicine, and Radiation Oncology (MNT), Odette Cancer Centre, Department of Pathology (JMB) and Neurosurgery (MF), Sunnybrook Health Sciences Centre, Toronto, Ontario; Departments of Pathology (MCG), Neurosurgery (MCG, RFD), Brain Tumour Research Centre, Montreal Neurological Insititute and Hospital, McGill University, Montreal, Quebec, Canada

Received March 16, 2009. Final Revisions Submitted April 23, 2009. Correspondence to: Jiwon Oh, University of Toronto, c/o Office of Dr. James Perry Sunnybrook Health Sciences Centre, A402, 2075 Bayview Ave, Toronto, Ontario, M4N 3M5, Canada. 

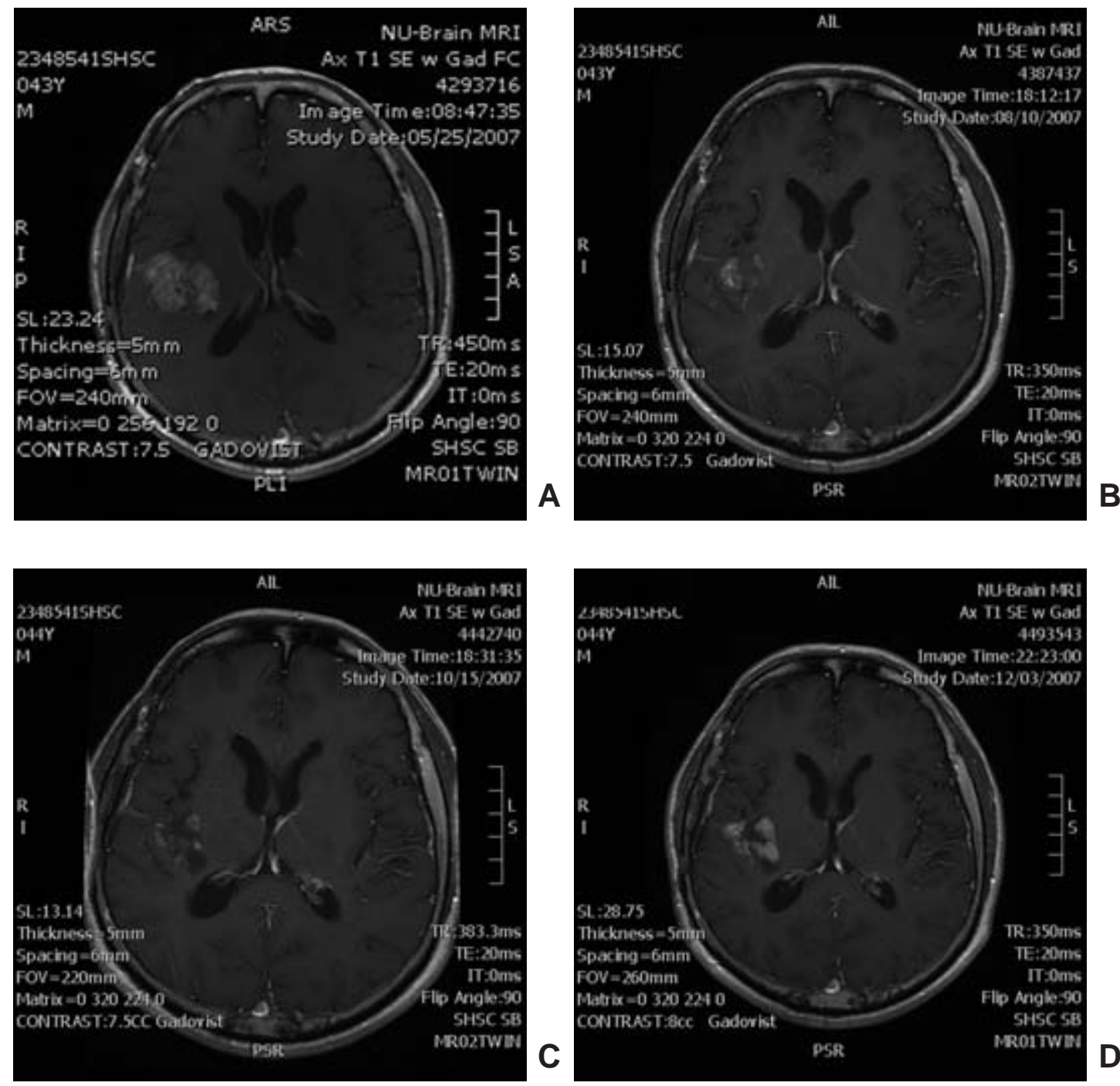

Figure 2: A) Recurrence of $3.5 \times 2.9 \mathrm{~cm}$ enhancing mass in right basal ganglia after 3 cycles of carboplatin/etoposide chemotherapy. B) Decrease in size, enhancement of mass in right basal ganglia after 3 cycles of temozolomide chemotherapy. C) Sustained response after 4 cycles of temozolomide chemotherapy. D) Tumor recurrence after 6 cycles of temozolomide chemotherapy.

cavity. A thallium scan confirmed avid uptake in this area, which was suggestive of recurrent disease, as opposed to radiation necrosis. He was thus started on adjuvant carboplatin/etoposide chemotherapy (carboplatin once/month, continuous two-week cycles of etoposide). He tolerated this regimen well, and had no significant clinical or hematological side effects. Unfortunately, after completing three cycles of this regimen, a repeat MRI-head showed evidence of tumour progression, with an increase in size of the enhancing mass from $2.2 \times 1.9 \mathrm{~cm}$ to $3.5 \times 2.9 \mathrm{~cm}$ (Figure 2A).

In the absence of any distinct guidelines regarding treatment of this rare tumour, his chemotherapy regimen was then switched to temozolomide at $300 \mathrm{mg}$ per day $\left(150 \mathrm{mg} / \mathrm{m}^{2}\right)$ in conventional $5 / 28$ day cycles. He tolerated the medication quite well with no significant clinical or hematological side effects. He was seen in repeat consultation by the radiation oncologist, who determined that he was not a candidate for repeat radiotherapy at that time, as he had recently received a course of radical radiotherapy. $\mathrm{He}$ was also seen in follow-up by the neurosurgeon involved in his care, and surgery was to be considered only as a last-resort option, if he did not respond to medical management.

An MRI-head obtained after the patient completed two cycles of temozolomide showed clear evidence of a response (Figure 2B). The enhancing mass had diminished significantly in size to $1 \mathrm{~cm} \times 1 \mathrm{~cm}$, with only a small amount of residual enhancing tissue within the right frontal/temporal lobe. After completing four cycles of temozolomide, the response was sustained with no radiographic evidence of change in comparison to the previous scan (Figure 2C). The patient remained clinically well with no significant side effects from the medication. 
Given his positive response to temozolomide, the activity of the DNA repair enzyme MGMT (O6-methylguanine-DNA methyltransferase) was assessed in our patient's pathological specimen by assessing for methylation of the promoter region, which functionally acts to silence the gene. This was carried out by MSP (methylation specific polymerase chain reaction [PCR]) following bisulfate treatment of DNA extracted from formalin paraffin embedded tissue. Interestingly, this patient's MGMT promoter was methylated, which is in keeping with his response to temozolomide (Figure 3).

Unfortunately, after six cycles of temozolomide chemotherapy, there was evidence of recurrence on MRI (Figure 2D). At this point, his chemotherapy was changed to daily temozolomide at $100 \mathrm{mg}$ daily $\left(50 \mathrm{mg} / \mathrm{m}^{2}\right)$ as "rescue" therapy, with no improvement at two months. He subsequently underwent further tumour resection, and was started on CCNU chemotherapy at $200 \mathrm{mg}$, given in six week cycles. After one cycle of CCNU, there was stabilization of his tumour. However, after four cycles of treatment, he had clear evidence of tumour recurrence with significant clinical deterioration, and died within a few months.

\section{Discussion}

Supratentorial PNET in adults is an uncommon entity. A previous study reports only 57 cases of supratentorial PNET in adults in the existing literature ${ }^{2}$. Due to its low incidence, to date, the optimal medical management of this tumour remains unknown.

Current management initially consists of total resection, if possible, followed by radiotherapy. Due to the propensity of PNET to disseminate in CSF, radiation of the entire neuroaxis is usually recommended ${ }^{2}$. The prognosis of PNET has improved significantly with radiotherapy, and long-term survival in adults is reported to be $50-60 \%$ at five years ${ }^{5}$. Adjuvant chemotherapy is utilized in some centres, but the available data is unclear about its role ${ }^{4}$. Agents that have been used in previous reports include CCNU, vincristine, cisplatinum, procarbazine, etoposide,

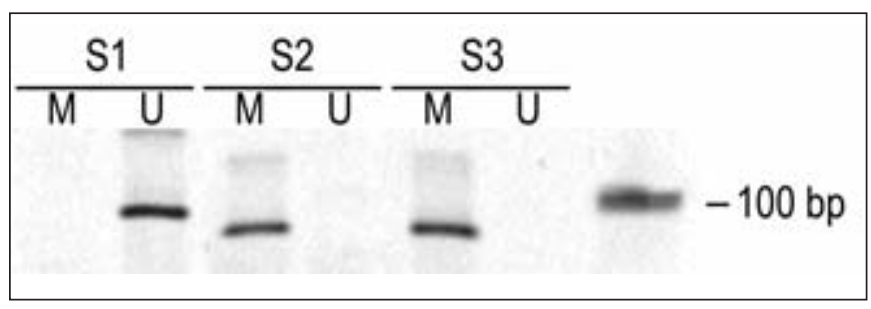

Figure 3: Methylation specific PCR of MGMT promoter. S1: placental DNA shows an unmethylated band (90bp). S2: Hela cell DNA shows a methylated band. S3: index case shows a methylated band (70bp). Last lane represents $100 \mathrm{bp}$ DNA ladder.

cyclophosphamide, and carboplatin ${ }^{4}$. There have been variable response rates reported with these agents.

Temozolomide is a newer alkylating chemotherapeutic agent used in the treatment of glioblastoma multiforme and anaplastic astrocytoma. This agent is generally well-tolerated, with the main major side effects being nausea and myelosuppression.

In the existing literature, temozolomide use in the context of adult PNET recurrence has only been reported in a handful of previous cases (Table). One case described temozolomide use in recurrence of a previously diagnosed childhood $\mathrm{PNET}^{1}$. The second case is that of recurrent adult supratentorial PNET ${ }^{4}$. Both cases reported showed good responses with the use of temozolomide. Two other cases describe initial responses followed by subsequent relapses of adults with recurrent PNET treated with temozolomide chemotherapy ${ }^{6,7}$. Finally, two previous case series of adult PNET patients mention three cases that demonstrated a response with temozolomide ${ }^{8,9}$.

Our case was that of an aggressive supratentorial PNET that failed chemotherapy with the more conventional agents: carboplatin and etoposide. With temozolomide, however, the tumour showed a striking radiographic response that was durable

Table: Summary of Reported Cases of Adult PNET with Response to Temozolomide

\begin{tabular}{|c|c|c|c|c|}
\hline $\begin{array}{l}\text { Authors / Publication } \\
\text { Year }\end{array}$ & Age / Sex & $\begin{array}{l}\text { Initial PNET } \\
\text { location }\end{array}$ & $\begin{array}{l}\text { Recurrent PNET } \\
\text { location }\end{array}$ & Response to Temozolomide \\
\hline $\begin{array}{l}\text { O'Reilly et al, } 1993^{9} \\
\text { (Case Series) }\end{array}$ & $\begin{array}{l}1 \text { patient - } \\
\text { demographics n/a }\end{array}$ & $\mathrm{n} / \mathrm{a}$ & Bony metastases & Complete response, sustained for 6 months \\
\hline Rao et al, $2001^{1}$ & 31 , female & Pineal mass & $\begin{array}{l}\text { Dural mass at L4-L5, C6- } \\
\text { C7, cerebellum }\end{array}$ & Complete response \\
\hline $\begin{array}{l}\text { Herrlinger et al, } 2005^{8} \\
\text { (Case Series) }\end{array}$ & $\begin{array}{l}2 \text { patients - } \\
\text { demographics n/a }\end{array}$ & $\mathrm{n} / \mathrm{a}$ & $\mathrm{n} / \mathrm{a}$ & $\begin{array}{l}\text { Patient 1: Complete response, sustained for } 5 \text { months } \\
\text { Patient 2: Complete response, sustained for } 12 \text { months }\end{array}$ \\
\hline Terheggen et al, $2007^{4}$ & 26 , male & Right parietal lobe & $\begin{array}{l}\text { Liver, bone marrow, right } \\
\text { parietal lobe }\end{array}$ & Complete response \\
\hline Durando et al, $2007^{6}$ & 55 , male & $\begin{array}{l}\text { Left cerebellar } \\
\text { hemisphere }\end{array}$ & Right frontal parenchyma & Partial response, sustained for 9 months \\
\hline Poelen et al, $2007^{7}$ & 26 , female & Tentorium & $\begin{array}{l}\text { Posterior fossa, thoracic } \\
\text { and cervical cord, near } \\
\text { lateral ventricles }\end{array}$ & Partial response, sustained for 9 months \\
\hline Present Case & 42 , male & Right basal ganglia & Right basal ganglia & Partial response, sustained for 6 months \\
\hline
\end{tabular}


for six months. Our patient tolerated this medication well with no significant side effects.

Our patient's pathological specimen had a methylated MGMT promoter, which functionally acts to silence the DNA repair gene. The MGMT promoter methylation has been shown to predict a beneficial response to temozolomide in patients with glioblastoma multiforme $(\mathrm{GBM})^{10}$, and may be predictive of response in other tumors as well. A recent report describes an aggressive pituitary tumor that responded to temozolomide chemotherapy. On immunohistochemistry, this tumor showed lack of MGMT staining, while another pituitary tumor unresponsive to temozolomide showed high expression of MGMT $^{11}$. In the existing literature, to our knowledge, MGMT methylation status has never been reported in the context of adult supratentorial PNETs treated with temozolomide. Our case is unique in that we have documented a clear clinical response of an adult-onset supratentorial PNET to temozolomide which was durable for six months, together with evidence of a methylated MGMT promoter region, which is in keeping with the literature for MGMT methylation in GBM.

This case adds to the small body of literature regarding the medical management of adult supratentorial PNET. Clearly, further investigations are needed prior to establishing guidelines regarding optimal medical management of this rare tumour. Future studies looking at MGMT methylation status in PNET in relation to temozolomide responsiveness may be helpful to further explore the utility of temozolomide in this tumour. In the absence of established guidelines, however, given the tolerability and ease of administration of temozolomide, this agent should be considered as an option for chemotherapy in patients with aggressive PNET. Furthermore, our case illustrates that the methylation status of MGMT promoter may be useful in predicting a response to temozolomide in these patients.

\section{ACKNOWLEDGEMENTS}

Dr. James R. Perry is the Crolla Family Chair in NeuroOncology at the University of Toronto. Dr. Rolando F. Del Maestro is the William Feindel Chair In Neuro-Oncology at McGill University.

\section{REFERENCES}

1. Rao RD, Robins HI, Mehta MP. Late recurrence of a primitive neuro-ectodermal tumor. Oncology. 2001;61:189-91.

2. Ohba S, Yoshida K, Hirose Y, Ikeda E, Kawase T. A supratentorial primitive neuroectodermal tumor in an adult: a case report and review of the literature. J Neurooncol. 2008; 86(2):217-24.

3. Kim DG, Lee DY, Paek SH, Chi JG, Choe G, Jung HW. Supratentorial primitive neuroectodermal tumors in adults. J Neurooncol. 2002;60:43-52.

4. Terheggen F, Troost D, Majoie CB, Leenstra S, Richel DJ. Local recurrence and distant metastasis of supratentorial primitive neuro-ectodermal tumor in an adult patient successfully treated with intensive induction chemotherapy and maintenance temozolomide. J Neurooncol. 2007;82:113-6.

5. Brandes AA, Palmisano V, Monfardini S. Medulloblastoma in adults: clinical characteristics and treatment. Cancer Treat Rev. 1999;25:3-12.

6. Durando X, Thivat E, Gilliot O, Irthum B, Verrelle P, Vincent C, et al. Temozolomide treatment of an adult with a relapsing medulloblastoma. Cancer Invest. 2007;25:470-5.

7. Poelen J, Bernsen HJ, Prick MJ. Metastatic medulloblastoma in an adult; treatment with temozolomide. Acta Neurol Belg. 2007; 107:51-4

8. Herrlinger U, Steinbrecher A, Rieger J, Hau P, Kortmann RD, Meyermann R, et al. Adult medulloblastoma: prognostic factors and response to therapy at diagnosis and at relapse. J Neurol. 2005:252:291-9.

9. O'Reilly SM, Newlands ES, Glaser MG, Brampton M, RiceEdwards JM, Illingworth RD, et al. Temozolomide: A new oral cytotoxic chemotherapeutic agent with promising activity against primary brain tumours. Eur J Cancer. 1993;29A(7): 940-2.

10. Hegi ME, Diserens AC, Gorlia T, Hamou MF, de Tribolet N, Weller $\mathrm{M}$, et al. MGMT gene silencing and benefit from temozolomide in glioblastoma. N Engl J Med. 2005;352:997-1003.

11. Kovacs K, Scheithauer BW, Lombardero M, McLendon RE, Syro LV, Uribe $\mathrm{H}$, et al. MGMT immunoexpression predicts responsiveness of pituitary tumors to temozolomide therapy. Acta Neuropathol. 2008;115:261-2. 